\title{
Spread of infection with time-delay activation in a small world
}

\author{
P. A. Golovinski \\ Physics Research Laboratory, Voronezh State Technical University, Voronezh, Russia \\ E-mail: golovinski@bk.ru
}

\begin{abstract}
A model of the spread of viruses in a network of cities is considered, taking into account a delay caused by the long incubation period of the viruses. The usual infection spread and dynamics with a delay are compared. A temporary asymmetry of the infection spread has been identified, when the time of the pandemic developing significantly exceeds the time for its completion. Numerical simulations of the spread of viruses in a network of interconnected large and small cities were carried out, and pandemic features in a small world were revealed, including the possibility of re-infection of the megalopolises.
\end{abstract}

Keywords: viruses, spread, COVID-19, pandemic, delay, city, network

\section{Introduction}

The spread of viruses (SV) in systems with a network structure and the emergence of pandemics have fundamental scientific interest and are the vital problems for natural biological populations, the human community, as well as for artificial computer systems. The danger of global pandemics gives particular urgency to these problems, and the COVID19 pandemic made their importance even more clear.

The direct experiment in such complex systems is practically impossible, therefore, in addition to studying and analyzing specific cases, there remains the way of a rather limited laboratory and alternative mathematical modeling of pandemics. Due to the safety and speed of assessing the development of situations, one of the most power tools for studying the emergence, growth, spread and extinction of pandemics is their mathematical modeling and computer simulation.

The simplest model of SV refers to a homogeneous system in which the spread rate depends on the number of infected carriers and the number of individuals not yet infected. SV in such a system is wave-like and is described by the Fisher-Kolmogorov-Petrovsky-Piskunov equation [1, 2]. However, many natural, socio-economic and artificial technical systems are highly spatially heterogeneous, which greatly complicates the analysis and understanding the processes of SV in them [3, 4].

One of the most important factors influencing the understanding and control of pandemics is some time delay of the process, when a certain time elapses from the moment of infection to the moment of active manifestation of the virus, after which the individual becomes a source of infection $[5,6]$. The effects of delay mask the causal relationships in the dynamics of $\mathrm{SV}$, and make wrong any linear prediction, even in the short term.

The outbreak of the global pandemic of the COVID-19, which is a global medical, political, organizational, moral, economic and intellectual challenge to humanity, has stimulated not only an intensified search for drugs, but also a simulation of the mechanisms of SV in the human population [7-10], as a better understanding mechanisms for the evolution of pandemics allows us to make optimal decisions when dealing with them. The simplest step-by-step model of the propagation of the network lesions, based on representation of a network as a graph, shows the critical dependence of the network resistance to the spread of viraltype lesions on the degree of its connectivity. The graphbased theory matrix analysis reveals the extreme vulnerability of the global networks. 
The key goal of our efforts is to obtain a decision-making tool based on the choice of the best option, within the framework of the system analysis paradigm, when to find exact solution of the problem is impossible, but a number of characteristic scenarios can be distinguished. In this paper, we propose dynamic network mathematical model, as a generalization of available models and approaches, and present the results of the study of few artificially constructed examples that demonstrate possible scenarios for the evolution of a pandemic.

\section{The mathematical model of the spread of viruses}

The simplest SIR (susceptible, infection, recovered) model of the spread of viral infection in the population is described by a system of three differential equations [11]. We apply this model to a set of interconnected cities between which a certain proportion of infected individuals are exchanged [12] with conservation the general balance of the subpopulations.

We denote the number of uninfected individuals in a given city $S_{k}$, the number of infected $I_{k}$, and the number of those who left the process as a result of a cure with stable immunity or who died $R_{k}$. The system of balance equations describing this process has the form

$$
\begin{aligned}
& \frac{d S_{k}}{d t}=-\beta_{k}(t) \frac{S_{k} I_{k}(t-\tau)}{N_{k}}-\sum_{j \neq k} \lambda_{k j}(t) I_{j}\left(t-\tau_{k j}\right), \\
& \frac{d I_{k}}{d t}=\beta_{k}(t) \frac{S_{k} I_{k}(t-\tau)}{N_{k}}-g_{k}(t) I_{k}(t-T) \\
& +\sum_{j \neq k} \lambda_{k j}(t) I_{j}\left(t-\tau_{k j}\right), \\
& \frac{d R_{k}}{d t}=g_{k}(t) I_{k}(t-T) .
\end{aligned}
$$

In this system of equations, the term $-g_{k} I_{k}(t-\tau)$ in the right-hand side of the second equation takes into account the elimination of infected virulent individuals from SV due to isolation effect. The last term in the right-hand side of the second equation is responsible for the transport of viruses by traffic flows. In this way, our model is distinguished from previous models by the explicit consideration of delays. In these equations the total number of individuals of all types in the $k$-th subpopulation $N_{k}=S_{k}+I_{k}+R_{k}$ is conserved; $\tau$ is the incubation period of the virus, causing a delay in the reproduction of the virus counting from the time of infection; $\tau_{k j}$ is a delay time due to the transport of infected between cities; $T$ is the average delay time from infection to isolation; $\beta_{k}(t)$ is the infection rate; $g_{k}(t)$ is the coefficient of isolation of existing patients; $\lambda_{k j}(t)$ is the coefficient characterizing the rate of transfer of infected from the $j$ - th city to the city with number $k$.
Coefficients $\beta_{k}(t)$ can be determined by knowing the number of infected individuals per one day. In the same way, it is possible to determine coefficients $g_{k}(t)$ through the number of people who were isolated from the number of those who became infected in a unit time interval, taking into account the additional shift due to the time of the disease detection. Coefficients $\lambda_{k j}(t)$ can be extracted from the traffic statistics. All the coefficients are individual for each node (city) and, in general case, may depend on time.

We suppose that the number of infected individuals arriving from another city is proportional to the number of infected individuals in the source. This means the absence of dynamics of change during transportation, i.e. short lag times typical for air travel. In this case, we can simply put the delay time equal to zero. Otherwise, when considering a vessel or a long-distance train as a vehicle, it is necessary to modify the system of equations (1), and consider more completely the changes with the health of passengers during a long travel time.

\section{Numerical examples and discussions}

The system of equations (1) has a simple structure, but it is nonlinear, and analytically unsolved although its numerical solution is quite simple. Observing the testable symptoms of the incidence, we do not see today picture, but situation several days before due to the latent period of infection. For further numerical simulations, we take delay constants $\tau=5, \tau_{k j}=0, T=8$ days.

As a first step, we demonstrate the dynamics of SV in a particular city, described by the couple of equations

$$
\begin{aligned}
& \frac{d S}{d t}=-\beta \frac{S I(t-\tau)}{N}, \\
& \frac{d I}{d t}=\beta \frac{S I(t-\tau)}{N}-g I(t-T)
\end{aligned}
$$

with initial conditions $I(0)=1, S(0)=N$. In the absence of the infection transfer from other subpopulations, it is constructive to introduce new variables $s=S / N, i=I / N$, and we get

$$
\begin{aligned}
& \frac{d s}{d t}=-\beta s i(t-\tau), \\
& \frac{d i}{d t}=\beta s i(t-\tau)-g i(t-T)
\end{aligned}
$$

with the initial conditions $i(0)=1 / N, s(0)=1$. Thus, our equations demonstrate self-similarity, with the exception of the influence of the initial conditions. They differ from the standard SIR equations by taking into account two delay effects: the duration of the incubation period when the individual is infected but not yet a source of viruses, and the average duration from infection to isolation. 
Figure 1 shows the result of numerical simulation of the solution of equations (3) for a city with population of 1 million inhabitants, without delays (two left-shifted lower magnitude curves) and with delays (right curves) for various coefficients of infection and isolation.

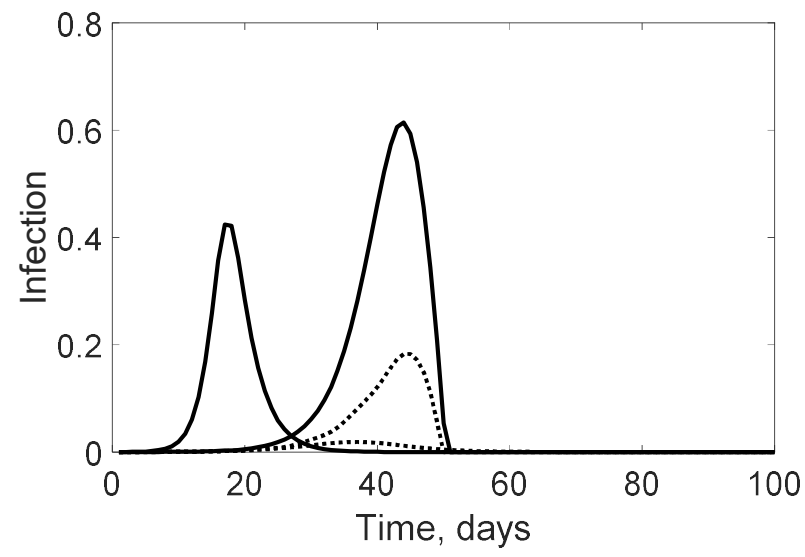

Figure 1. The spontaneous dynamics of SV for $\beta=1.4, g=0.3$ (solid lines), and SV with restrictions ( $\beta=1.1, g=0.9$, dot lines).

Simulation with a single individual initial infection demonstrates the effects of delay significantly worsen the picture of a pandemic. Comparison of the spontaneous dynamics of SV and the dynamics with the restriction on contacts and isolation of infected people shows the effectiveness of quarantine measures.

The existing systems of cities connecting by traffic streams complicate the picture of SV [13]. We consider a couple artificially constructed examples demonstrating the different dynamics of SV between cities connected by traffic flows. As options, we look the spread of viruses in fully connected systems. We take two groups of cities: cities with a population of one million people and cities with a population of two hundred thousand people. This allows us to analyze the effect of convective transport of infection on the heterogeneous spread of viruses. The first two equations of the system (1) are solved independently of the third. Given the approximations made, we obtain a closed system of equations

$$
\begin{aligned}
& \frac{d S_{k}}{d t}=-\beta \frac{S_{k} I_{k}(t-\tau)}{N_{k}}-\lambda \sum_{j \neq k} I_{j}(t), \\
& \frac{d I_{k}}{d t}=\beta \frac{S_{k} I_{k}(t-\tau)}{N_{k}}-g I_{k}(t-T)+\lambda \sum_{j \neq k} I_{j}(t),
\end{aligned}
$$

where to further simplify the analysis, we assumed that $g_{k}(t)=g, \beta_{k}(t)=\beta, \lambda_{k j}(t)=\lambda$, i.e., the rate of all the processes are the same across the network of cities and independent on time. This extreme simplification will make it possible, by intentionally reducing the detail of the model, to understand the influence of factors of different origin outside their inevitable distribution over magnitude in the real systems. We take $\lambda=1 / 1000$ universal for all transport links between cities. The parameters taken by us are of an evaluative model nature and are not reliable for reproducing real-world situations.

For analysis, we separately display the percentage dynamics of SV for groups of large and small cities. The results of modeling SV in a full-connected system with one millionth city and five small cities, having the first single infection in the largest of them, are shown in figure 2 when $\beta=1.4, g=0.3$ (higher magnitudes), and when subject to additional restrictions on the infection rate and isolation of the population ( $\beta=1.1, g=0.9$, lower magnitudes).

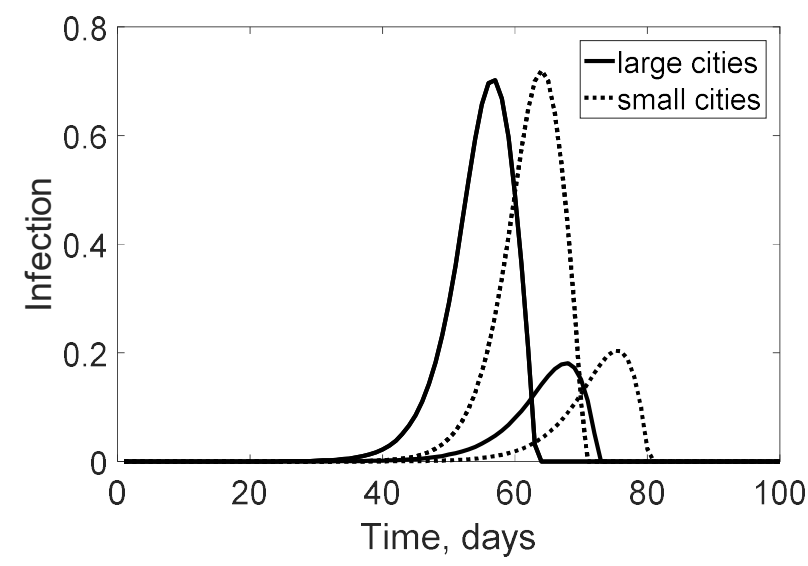

Figure 2. The dynamics of SV in a small network of 6 fully connected cities.

The results of numerical simulation of SV in a fully connected system of five large cities and fifty small cities with the first single infection in a large city are shown in figure 3: when $\beta=1.4, g=0.3$ (higher magnitudes), and under conditions of restriction of the people moving and isolation of the infected population $(\beta=1.1, g=0.9$, lower magnitudes).

The main feature of the infection dynamics in such a small world is the non-uniformity of the infection growth in the large cities and a group of small cities. When viruses spread in a system of connected cities, starting from a large city, an additional delay effect arises due to the time spent on the transfer of pathogens from city to city. Another feature of the dynamics of a pandemic in a network with large cities surrounded by a number of small cities is the occurrence of a nonmonotonic in time growth rate of infections and even possible local intervals of the recession, which can be misleading, causing unreasonable assumptions about the near end of the pandemic and the premature removal of restrictive measures. This non-uniformity is due to the role of the environment as a reservoir for re-infection. The second wave of infection can be avoided by introducing short-term 
restrictions on entry into large cities during the peak phase of infection in small cities.

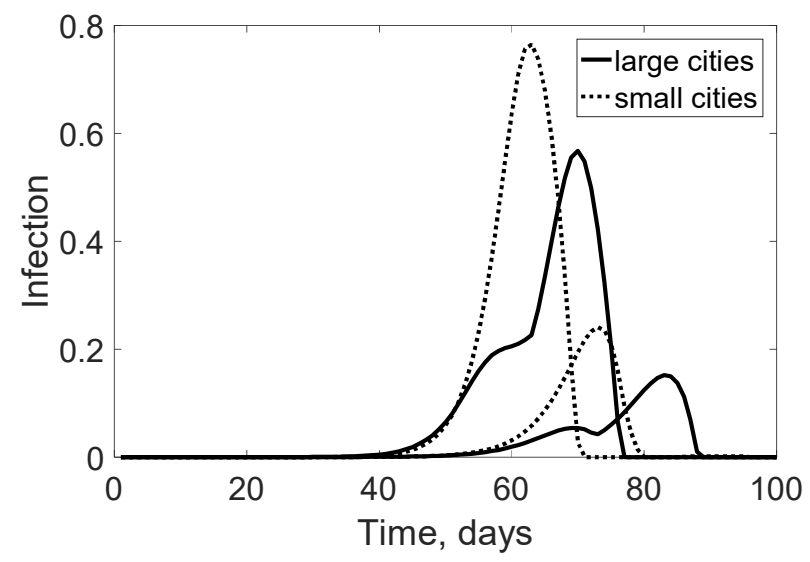

Figure 3. The dynamics of SV in a large network of 55 connected cities.

The comparative dynamics in two different networks with one large city and a number of large cities demonstrates the inversion effect of the infection order for large and small cities for these two substantially different cases.

\section{Conclusions}

The incubation delay of several days leads to substantial increasing duration of a pandemic, stretching it from the more usual monthly period up to the $90-100$ days interval in a separate megapolis in a network of connected cities. The dynamics of infection is asymmetrical with respect to the peak shape. For the fixed kinetic coefficients the dynamics simulation with time-delay effects predicts the increasing of infection takes longer and more smoothly, compared with the completion phase of the spread of infection. Reaching a constant growth rate of infected people indicates that the peak of the pandemic is approaching, but only in the absence of surrounding infection reservoir.

The best scenario for combating a pandemic depends on the economic power of society or its willingness to endure restrictions and hardships, especially in large metropolitans. The number of lives saved and the degradation of an idle economy are closely related together. However, decreasing the rate of virus infection spread due to social isolation separately without strict isolation measures does not produce a significant inhibitory effect.

Our modification of SIR consider the nonlocality in time of the processes in a simplified form using delay constants, while a more detailed description can be obtained with integral operators that take into account memory effects. The development of such more sophisticated model is hindered not only by theoretical difficulties, but also by the vagueness of the practical availability of determining its parameters.
However, the general conclusion about the feasibility of tough measures restricting SV with a long incubation phase, using combined social distance and isolation of infected people, our model demonstrates with high visibility. We note that the simulation of real scenarios requires the use of specific data on the number of cities, transport activity, as well as the infection and isolation rate constants.

\section{References}

[1] Kolmogorov A., Petrovskii I. and Piskunov N. 1991 In V. M. Tikhomirov, editor, Selected Works of A. N. Kolmogorov I, Kluwer, Dordrecht, 248

[2] Fisher R. A. 1937 Ann. Eugenics 7353

[3] Longini I. M. 1988 Mathematical Biosciences 90367

[4] Nokkaew A., Modchang C., Amornsamankul S. et al.. 2017 Adv. Differ. Equ. 288

[5] Liu Junli, Wu Jianhong and Zhou Yicang. 2008 Rocky Mountain J. Math. 381525

[6] Misra A. K., Mishra S. N., Pathak A. L., Misra P., and Naresh R. 2012 Applied Mathematics and Computation 21811547

[7] Chen T., Rui J., Wang Q. et al. 2020 Infect. Dis. Poverty 924

[8] Qianying Lin et al.. 2020 International Journal of Infectious Diseases 93211

[9] BiaoTang et al.. 2020 Infectious Disease Modelling 524

[10] Wu J.T., Leung K. and Leung G. M. 2020 Lancet 395689

[11] Chen D. 2015 In Analyzing and Modeling Spatial and Temporal Dynamics of Infection Diseases. Book Editor(s): Dongmei Chen, Bernard Moulin, Jianhong Wu, John Wiley \& Sons, Hoboken, 19

[12] Arino J., Hu, W.H., Khan, et al. 2012 Canadian Applied Mathematics Quarterly 19125

[13] Bian L. and Liebner D. 2007 Transactions in GIS 11155 